\title{
VALIDAÇÃO DA CALIBRAÇÃO DE UMA BALANÇA EXTERNA DE TÚNEL DE VENTO*
}

\author{
${ }^{1}$ Maria L.C.C. Reis ${ }^{+}$ \\ ${ }^{2}$ Wilson J. Vieira ${ }^{+}$ \\ ${ }^{3}$ Itamar M. Barbosa ${ }^{\#}$ \\ ${ }^{4}$ Olympio A.F. Mello ${ }^{+}$ \\ ${ }^{5}$ Luciano A. Santos ${ }^{+}$
}

Resumo: Balanças aerodinâmicas são empregadas em túneis de vento para medir as cargas atuantes em modelos sob teste. Uma metodologia foi desenvolvida para a avaliação da incerteza de calibração da balança externa do Túnel de Vento Subsônico N. ${ }^{\circ}$ 2, do Centro Técnico Aeroespacial de São José dos Campos. A fim de obter um modelo adequado para fornecer as cargas aerodinâmicas a partir das medições das células de carga da balança, anteriormente ao ensaio, realize-se uma calibração aplicando-se pesos na balança através de um sistema de cabos e roldanas. Dois procedimentos são empregados para relacionar as leituras das células de carga às cargas aerodinâmicas: ajuste de curva e Redes Neurais. A modelagem matemática para a regressão por mínimos quadrados é multivariada. A classe de Redes Neurais usada é a rede Multy-Layer Perceptrons (MLP) ou Perceptrons de Multicamadas. A análise de erro inclui o emprego da lei geral de Propagação de Incerteza à curva resultante da regressão multivariada e o método de Simulação Monte Carlo. Uma comparação dos métodos utilizados é destacada neste trabalho.

Palavras-chave: Incertezas, Calibração, Ensaios, Redes Neurais Artificiais.

Abstract: Aerodynmic balances are employed in wind tunnels to measure the loads acting on the model under test. A methodology has been developed for the assessment of the calibration uncertainty of the external balance at the Subsonic Wind Tunnel No. 2 (TA-2), at the Aerodynamic Laboratory, (ASA-L), of the Centro Técnico Aeroespacial, (CTA), Brazil. In order to obtain a suitable model to provide aerodynamic loads from the balance load cell measurements, a balance calibration is performed prior to the tests, applying loads to the balance through a system of cables and pulleys. Two approaches are employed to relate the load cell readings and the areodynamic loads: curve fitting and neural networks. The mathematical model for the least-squares regression is the multivariate one. The class of neural networks used is the multilayer feedforward networks, commonly referred to as multilayer perceptrons. The error analysis includes the employment of the general law of

\footnotetext{
${ }^{1}$ mluisareis@iae.cta.br, ${ }^{2}$ wjvieira@ieav.cta.br, ${ }^{3}$ itamar.barbosa@ifi.cta.br, ${ }^{4}$ oamello@iae.cta.br,

${ }^{5}$ lucianosantos@iae.cta.br,

+ Centro Técnico Aeroespacial

Pça. Marechal Eduardo Gomes N 50 São José dos Campos - SP- Brasil - CP6001 - CEP12228-901

* Escola Politécnica da Universidade de São Paulo

Cidade Universitária - AV. Professor Luciano Gualberto - São Paulo, SP, CEP 05508-900 - Brasil

* Este artigo foi apresentado na $24^{\mathrm{a}}$ Conferência de Tecnologia de Medições Aerodinâmicas e Ensaios de Solo.
} 
propagation of uncertainty to the curve resulting from the multivariate regression and the method of Monte Carlo Simulation. A comparison of the approaches used is outlined in this paper.

Keywords: Uncertainty, Calibration, Tests, Artificial Neural Network.

\section{INTRODUÇÃO}

Laboratórios de Ensaios no Solo por todo o mundo se preocupam com a padronização da estimativa de incerteza das grandezas medidas em ensaios aerodinâmicos.

A equipe do Laboratório Aerodinâmico (ASA-L), seguindo esta tendência, desenvolveu vários projetos para melhorar a qualidade de seus serviços. Parte do processo para obter os requisitos para competência do laboratório inclui a validação de metodologias empregadas nos ensaios aerodinâmicos. Fornecer dados de ensaio no solo confiáveis em curto período de tempo é a chave para ajudar nossos clientes a manter sua competitividade no mercado aeronáutico internacional.

Estudos relacionados à análise de incerteza no túnel de vento subsônico TA-2 resultaram em vários documentos ${ }^{1,}$ 2, 3, 4, 5 . O objetivo deste estudo é validar a metodologia de calibração da balança externa incluindo a avaliação da incerteza das cargas aerodinâmicas medidas.

Os valores das cargas aerodinâmicas estimadas através do ajuste de curva são comparados àqueles obtidos a partir da aplicação das Redes Neurais.

A validação da estimativa da incerteza das cargas é realizada pelo procedimento numérico conhecido com método de Simulação Monte Carlo.

\section{PROPAGAÇÃO DA INCERTEZA}

A metodologia de avaliação de incerteza adotado está descrita no Guia (GUM), $1995^{6}$.

A incerteza padrão da estimativa $y$ do mensurando $Y$ é a combinação das incertezas padrão das estimativas de entrada, $x_{1}, x_{2}, \ldots, x_{N}$, suas covariâncias e a não linearidade da modelagem matemática, e é dada por:

$$
\begin{aligned}
& u_{c}^{2}(y)=\sum_{i=1}^{N}\left(\frac{\partial y}{\partial x_{i}}\right)^{2} u^{2}\left(x_{i}\right)+2 \sum_{i=1}^{N-1} \sum_{j=i+1}^{N} \frac{\partial y}{\partial x_{i}} \frac{\partial y}{\partial x_{j}} u\left(x_{i}, x_{j}\right)+ \\
& +\sum_{i=1}^{N} \sum_{j=1}^{N}\left[\frac{1}{2}\left[\frac{\partial^{2} f}{\partial x_{i} \partial x_{j}}\right]^{2}+\frac{\partial f}{\partial x_{i}} \frac{\partial^{3} f}{\partial x_{i} \partial x_{j}^{2}}\right] u^{2}\left(x_{i}\right) u^{2}\left(x_{j}\right)
\end{aligned}
$$

\section{CALIBRAÇÃO DA BALANÇA DO TÚNEL DE VENTO}

A balança externa de seis componentes é usada para medir as cargas $F_{i}(i=1, \ldots, 6)$ atuantes no modelo durante o ensaio no túnel de vento; $F_{1}, F_{2}$ e $F_{3}$ denotam forças e $F_{4}, F_{5}$ e $F_{6}$ denotam momentos. A balança é prismática para forças e piramidal para momentos. Uma calibração da balança é realizada anteriormente aos ensaios para fornecer as cargas aerodinâmicas que atuam no modelo a ser ensaiado. A calibração é realizada a partir da aplicação de cargas à balança através de um sistema de cabos e roldanas. Um conjunto de aproximadamente 100 pesos de $10 \mathrm{~kg}$ é usado para aplicar as cargas de calibração.

Setenta e três combinações são usadas para cada ângulo de ataque. A Tabela 1 apresenta algumas combinações de carregamentos típicos. Os valores que representam as cargas aplicadas são oriundas da aplicação de pesos na cruz de calibração. Estes dados experimentais têm sido usados para avaliar e ilustrar a metodologia de avaliação da incerteza no TA-2. Os símbolos $F_{1}, F_{2}, F_{3}, F_{4}, F_{5}$ e $F_{6}$ são usados para as forças de arrasto, lateral e sustentação, e para os momentos de rolamento, arfagem e guinada, respectivamente. 
Tabela 1: Alguns carregamentos típicos para a calibração da balança. Unidade: newton para forças e newtonmetro para momentos.

\begin{tabular}{cccccc}
\hline & \multicolumn{5}{c}{ Nímern do Carregamento } \\
\cline { 2 - 6 } & 1 & 26 & 38 & 60 & 71 \\
\hline$F_{1}$ & 0 & 500 & 0 & 500 & 1000 \\
\hline$F_{7}$ & 0 & 0 & 800 & 0 & 0 \\
\hline$F_{2}$ & -2400 & 0 & 0 & 0 & 2400 \\
\hline$F_{1}$ & 0 & -300 & 0 & 0 & 0 \\
\hline$F_{5}$ & -300 & 0 & 0 & -300 & 0 \\
\hline$F_{6}$ & 0 & 0 & 240 & 0 & 0 \\
\hline
\end{tabular}

\section{MODELAGEM DA CALIBRAÇÃO}

Uma curva de calibração é ajustada para cada conjunto de 73 pontos $\left(F_{i}\right.$; $\left.S_{1}, S_{2}, S_{3}, S_{4}, S_{5}, S_{6}\right)_{k}, i=1, \ldots, 6$.

O modelo do ajuste é uma combinação linear de 27 funções de $S_{i}$ que são: $S_{1}, S_{2}$, $S_{3}, S_{4}, S_{5}, S_{6}, S_{1}^{2}, S_{1} S_{2}, S_{1} S_{3}, S_{1} S_{4}, S_{1} S_{5}$, $S_{1} S_{6}, S_{2}{ }^{2}, S_{2} S_{3}, S_{2} S_{4}, S_{2} S_{5}, S_{2} S_{6}, S_{3}{ }^{2}, S_{3} S_{4}$, $S_{3} S_{5}, S_{3} S_{6}, S_{4}{ }^{2}, S_{4} S_{5}, S_{4} S_{6}, S_{5}{ }^{2}, S_{5} S_{6}, S_{6}{ }^{2}$. O modelo, portanto, compreende vinte e sete parâmetros ajustáveis $a_{j}(j=1, \ldots, 27)$ para $F_{1}$, vinte e sete parâmetros ajustáveis $b_{j}$ para $F_{2}$, e assim por diante, até chegar aos parâmetros $f_{j}$ para $F_{6}$.

A equação matricial para o modelo multivariado (múltiplas entradas e saídas) é a seguinte:

$\left[\begin{array}{l}F_{1} \\ F_{2} \\ F_{3} \\ F_{4} \\ F_{5} \\ F_{6}\end{array}\right]_{6 \times 1}=\left[\begin{array}{cccc}a_{1} & a_{2} & \cdots & a_{27} \\ b_{1} & b_{2} & \cdots & b_{27} \\ \ddots & & & \\ & \ddots & & \\ & & \ddots & \\ f_{1} & f_{2} & \cdots & f_{27}\end{array}\right]_{6 \times 27}\left[\begin{array}{c}S_{1} \\ S_{2} \\ \vdots \\ S_{6} \\ S_{1}^{2} \\ S_{1} S_{2} \\ S_{1} S_{3} \\ \vdots \\ S_{5} S_{6} \\ S_{6}^{2}\end{array}\right]_{27 \times 1}$

$$
\begin{aligned}
& F_{1}(S)=a_{1} S_{1}+a_{2} S_{2}+a_{3} S_{3}+a_{4} S_{4}+a_{5} S_{5}+a_{6} S_{6}+ \\
& a_{7} S_{1}^{2}+a_{8} S_{1} S_{2}+a_{9} S_{1} S_{3}+a_{10} S_{1} S_{4}+a_{11} S_{1} S_{5}+a_{12} S_{1} S_{6}+ \\
& a_{13} S_{2}^{2}+a_{14} S_{2} S_{3}+a_{15} S_{2} S_{4}+a_{16} S_{2} S_{5}+a_{17} S_{2} S_{6}+a_{18} S_{3}^{2} \\
& +a_{19} S_{3} S_{4}+a_{20} S_{3} S_{5}+a_{21} S_{3} S_{6}+a_{22} S_{4}^{2}+a_{23} S_{4} S_{5}+ \\
& a_{24} S_{4} S_{6}+a_{25} S_{5}^{2}+a_{26} S_{5} S_{6}+a_{27} S_{6}^{2}
\end{aligned}
$$

A dependência do modelo em seus parâmetros $a_{j}, b_{j}, \ldots, f_{j}, \quad(j=1, \ldots, 27)$ é linear. As forces aplicadas $F_{i}$ e as leituras $S_{i}, \quad(i=1, \ldots, 6)$ são as grandezas de entrada da calibração. Os parâmetros $a_{j}$ $, b_{j}, \ldots, f_{j}$ obtidos no ajuste de curva, são os mensurandos (ou grandezas de saída) a serem determinados, e além de estimálos, é necessário também fornecer uma estimativa de suas variâncias e de suas covariâncias $^{6,7,8}$.

\section{INCERTEZAS NAS CARGAS AERODINÂMICAS}

A aplicação da lei de propagação de incerteza às equações que representam o modelo ajustado leva à incerteza padrão combinada dos valores $F_{i}$, estimados pelo ajuste de curva, para quaisquer $S_{i}$. Considerando a carga $F_{1}$, a incerteza combinada para $\mathrm{N}$ grandezas de entrada correlacionadas é fornecida aplicando a Equação (1) à Equação (3):

Os termos, $u^{2}\left(a_{j}\right)$ e $u\left(a_{j}, a_{l}\right)$, são as variâncias e as covariâncias dos parâmetros ajustados, $a_{j}$. Os termos $u^{2}\left(S_{i}\right)$ correspondem ao valor quadrático das incertezas de calibração, definidos nos certificados de calibração das células de carga.

A não linearidade não é incluída na Equação (4), pois sua contribuição para a incerteza é negligenciável ${ }^{5}$.

Por exemplo, os 73 pontos $\left(F_{1}\right.$; $\left.S_{1}, S_{2}, S_{3}, S_{4}, S_{5}, S_{6}\right)_{k}$ são ajustados ao modelo: 


$$
\begin{aligned}
& u_{c}^{2}\left(F_{1}\right)=\left(S_{1}\right)^{2} u^{2}\left(a_{1}\right)+\left(S_{2}\right)^{2} u^{2}\left(a_{2}\right)+\ldots \\
& +\left(S_{6}\right)^{2} u^{2}\left(a_{6}\right)+\ldots+\left(S_{6}\right)^{4} u^{2}\left(a_{27}\right)+\cdots \\
& +2\left[S_{1} S_{2} u\left(a_{1}, a_{2}\right)+S_{1} S_{3} u\left(a_{1}, a_{3}\right)+\cdots\right. \\
& \left.+S_{5} S_{6} S_{6}^{2} u\left(a_{26}, a_{27}\right)\right]+ \\
& +\left(a_{1}+2 a_{7} S_{1}+a_{8} S_{2}+a_{9} S_{3}+a_{10} S_{4}+\right. \\
& \left.+a_{11} S_{5}+a_{12} S_{6}\right)^{2} u^{2}\left(S_{1}\right)+\ldots \\
& +\left(a_{6}+a_{12} S_{1}+a_{17} S_{2}+a_{21} S_{3}+a_{24} S_{4}+\right. \\
& \left.+a_{26} S_{5}+2 a_{27} S_{6}\right)^{2} u^{2}\left(S_{6}\right)+ \\
& +\frac{1}{2}\left(2 a_{7}\right)^{2} u^{2}\left(S_{1}\right) u^{2}\left(S_{1}\right)+\cdots+ \\
& +\frac{1}{2}\left(2 a_{27}\right)^{2} u^{2}\left(S_{6}\right) u^{2}\left(S_{6}\right)
\end{aligned}
$$

\section{O Ajuste de Mínimos Quadrados}

$\mathrm{O}$ arranjo dos valores de força $F_{i}$ aplicada à cruz de calibração na matriz $[F]$ e as leituras das células de carga na matriz [S], resulta nos parâmetros $a_{j}$ da curva de calibração, via inversão de matriz ${ }^{5,7}$ :

$$
[a]=\left[[S]^{t}[S]\right]^{-1}[S]^{t}[F]
$$

[S] é chamada matriz de projeto e $[S]^{t}$ é sua transposta.

A matriz erro, $\left[[S]^{t}[S]\right]^{-1}$, fornece as variâncias, $u^{2}\left(a_{j}\right)$, e as covariâncias, $u\left(a_{j}, a_{l}\right)$, dos parâmetros ajustados, $a_{j}$.

De acordo com as referências 9 e 10, a determinação dos parâmetros $a_{j}$ via inversão de matriz não é recomendada. Portanto, um método mais estável numericamente, o algoritmo de fatoração ortogonal $Q R$, foi também usado. Este algoritmo consiste na fatoração da matriz de projeto $[S]$ em uma matriz ortogonal e uma matriz triangular.

\section{A REDE NEURAL}

Redes Neurais Artificiais são técnicas de inteligência computacional que podem ser consideradas capazes de resolver certas classes de problemas, entre eles a aproximação de funções, algumas vezes chamada de modelagem matemática.

A modelagem em questão refere-se à calibração da balança externa do TA-2.
Redes Neurais já tinham sido empregadas em ajustes de curva de calibração ${ }^{11}$.

O projeto das Redes Neurais Artificiais envolve os seguintes aspectos: o número de camadas, o número de neurônios em cada camada, a função de transferência, o algoritmo de aprendizado, os valores iniciais dos pesos sinápticos, uma medida do desempenho de aprendizagem, e o número de iterações na aprendizagem ${ }^{12}$.

A Rede Neural usada neste estudo possui uma camada de entrada formada pelas variáveis de entrada, uma camada oculta de neurônios e uma camada de neurônios de saída. Ela é conhecida como MultiLayer Perceptrons (MLP) e é considerada completamente conectada, uma vez que cada nó em cada camada da rede está conectado a todos os outros nós na camada próxima adjacente. As MLPs foram escolhidas porquê a modelagem matemática da calibração da balança externa é multivariada.

A arquitetura da rede usada é apresentada na Figura 1.

Trinta Redes Neurais foram usadas, onde o número $i$ de nós (variáveis) da camada de entrada e o número de nós da camada de saída são iguais a 6 , já o número $n$ de nós da camada oculta variaram de 1 a 30 . (Resumindo, Redes: 6- $n-6, n=1, . ., 30$ ). $\mathrm{O}$ algoritmo de aprendizagem utiliza o método de Levenberg- Maquardt ${ }^{13}$. O número de iterações de aprendizagem foi 500. Para inicialização, os valores dos pesos sinápticos foram 0.0001. A medida de desempenho de aprendizagem adotada foi a somatória de erros quadráticos ${ }^{14}$, $\Sigma e^{2}$, que consiste na diferença quadrática entre a resposta real da rede neural e a resposta desejada, somada sobre todo o conjunto de dados.

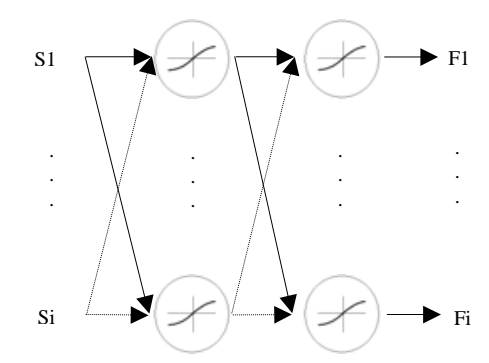

Figura 1: Arquitetura da rede neural. 
A função de ativação foi uma sigmoide do tipo:

$\varphi=\frac{2}{[1+\exp (-2 x)]}-1$

Em termos matemáticos, a função aproximada pela Rede Neural para cada variável $\mathrm{Fi}$ (forças e momentos) são descritos pela a seguinte equação:

$$
F_{i}=\varphi_{i}\left(\sum_{n=1}^{N} W_{2 i n} \varphi_{n}\left(\sum_{m=1}^{6} W_{1 n m} S_{m}\right)\right)
$$

$N=1,2, \ldots, 30$ (neste trabalho);

$F_{i}(i=1, \ldots, 6)$ são os sinais de saída;

$\varphi_{i}$ : função de ativação (ou função de transferência) dos neurônios da camada de saída;

$W_{\text {2inn }}$ : pesos sinápticos (ou parâmetros livres) dos neurônios da camada de saída; $\varphi_{n}$ : função de ativação (ou função de transferência) dos neurônios da camada oculta;

$W_{\text {1inn }}$ pesos sinápticos dos neurônios da camada oculta;

$S_{m}(m=1, \ldots, 6)$ são os sinais de entrada.

Há dois modos de operação da rede, o processo de aprendizagem e o processo de simulação. No primeiro, a resposta desejada (ou alvo de saída), representada pelos valores das cargas aplicadas à cruz de calibração, é fornecida à saída da rede. As leituras das células de carga são apresentadas à camada de entrada da rede. Ajustes são aplicados aos pesos sinápticos $W$ através de processo de aprendizagem iterativo. No segundo caso, as leituras das células de carga obtidas durante o ensaio são fornecidas à camada de entrada da rede e, a partir dos pesos $\mathrm{W}$ fixos determinados durante a fase de aprendizagem, obtém-se os valores das cargas aerodinâmicas correspondentes.

\section{SIMULAÇÃO PELO MÉTODO MONTE CARLO}

A aplicação da lei de propagação de incerteza à relação funcional entre $F$ e $S$
(Equação 3), cujo resultado é expresso na Equação (4), de acordo com a recomendação do $\mathrm{GUM}^{6}$, pode ser validada por uma técnica alternativa, o método de Simulação Monte Carlo (MCS), que foi adotado na ASA-L para avaliar as incertezas das cargas $F_{i}$ resultantes da curva de regressão.

O Método de Monte Carlo é uma técnica que propaga funções densidade de probabilidade $(p d f)$ ao invés de propagar incertezas das grandezas de entrada $X_{i}$ para fornecer a $p d f$ do mensurando $Y$.

Dada a distribuição, o valor médio e o desvio padrão das grandezas de entrada, um valor aleatório é gerado a partir da função distribuição para cada $X_{i}$. Estes valores são aplicados à relação funcional do experimento, que fornece a estimativa correspondente da grandeza de saída $Y$. A repetição deste processo por várias vezes resulta na estimativa do valor médio e da incerteza padrão do mensurando.

Para um modelo multivariado, onde há interdependência entre parâmetros, as entradas fornecidas ao método Monte Carlo são:

- os coeficientes da curva de calibração, ou seja, os parâmetros $a_{j}$ da Equação (3), suas incertezas $u\left(a_{j}\right)$ correspondentes e covariâncias $u\left(a_{j}, a_{l}\right)$;

- os valores das leituras das células de carga, $S_{i}$, as incertezas definidas em seus certificados de calibração, $u\left(S_{i}\right)$. Seus coeficientes de correlação são considerados iguais a um.

Em ambos conjuntos de dados, as distribuições de probabilidade assumidas são gaussianas.

\section{RESULTADOS E DISCUSSÕES}

Os parâmetros dos mínimos quadrados $a_{j}$, obtidos por dois meios, fatoração $Q R$ e inversão de matriz, são compatíveis, o que indica que não há instabilidades numéricas na inversão da matriz normal $[S]^{t}[S]$.

A contribuição para a não linearidade da modelagem matemática da balança (Equação 3) para a incerteza das cargas foi desprezada e portanto não considerada na incerteza combinada. A comparação 
entre a função de desempenho obtida a partir dos mínimos quadrados e da Rede Neural representada pela Equação (7), é mostrada na Figura 2 e na Tabela 2, em anexo. Nota-se que para um baixo número de neurônios na camada oculta (até $n<14$ ), a estimativa das cargas aerodinâmicas por curva de regressão é superior em comparação ao desempenho da Rede Neural. À medida que o número de neurônios ocultos aumenta, uma melhora ocorre no valor da função de desempenho da Rede Neural.

Os valores das incertezas, estimados de acordo com a recomendação do GUM, foram comparados aos obtidos pelo método de Monte Carlo e são apresentados na Tabela 3, em anexo. Os resultados do método de Monte Carlo são baseados em um milhão de histórias. A contribuição da interdependência entre os parâmetros ajustados $a_{j}$, expressa pelas covariâncias, foi também considerada.

\section{CONCLUSÕES}

De acordo com padronização metrológica internacional, a validação é mandatória em procedimentos de calibração e ensaio. A validação da calibração da balança externa é parte do processo de confiabilidade metrológica conduzido pelo TA-2. O processo inclui a exatidão da modelagem matemática e a avaliação da incerteza.

Foi realizada uma comparação entre o desempenho de uma modelagem polinomial e uma modelagem de Redes Neurais. Foi apresentado que a exatidão de ambos os métodos são compatíveis quando o número de neurônios na camada invisível está em torno de 14 . Entretanto, a discussão sobre modelagem é uma questão de necessidades do cliente, ou seja, os requisitos de exatidão de ensaio ditam a escolha da modelagem matemática.

A distribuição, média, desvio padrão e covariância dos parâmetros da curva de regressão e das células de carga foram fornecidos ao método de Monte Carlo, que confirmou as cargas aerodinâmicas e suas correspondentes incertezas.

\section{AGRADECIMENTOS}

Este trabalho foi financiado pela Fundação de Amparo à Pesquisa do Estado de São Paulo (FAPESP) sob no. 00/13769-0 e 04/01726-6.

\section{REFERÊNCIAS}

1 Mello O. A. F., Uyeno S., Sampaio O. S., Reis M. L. C. C. "Uncertainty Methodology at the Brazilian TA-2 Subsonic Wind Tunnel”, AIAA Paper 98-2716, AIAA 20th Advanced Measurement and Ground Testing Technology Conference, Albuquerque, New Mexico, USA, June 1998.

2 Reis M. L. C. C, Mello O. A. F., Uyeno S., Sampaio O. S. "A Method for Estimation of the Calibration Uncertainty of an External Six Component Wind Tunnel Balance”, AIAA Paper 2002-2793, $22^{\text {nd }}$ AIAA Aerodynamic Measurements and Ground Testing Conference, 24 - 26 June 2002, St. Louis, MO.

3 Reis M. L. C. C., Novaski O., Mello O. A. F., Sampaio O. S., "Evaluacion de la Incertidumbre de la Medicion en Tunel de Viento", [Evaluation of the Uncertainty in Measurement in a Wind Tunnel] Información Tecnológica, La Serena, Chile, Vol. 11, No. 6, 2000, pp. 151-160.

4 Reis M. L. C. C, 2000, “Expressão da Incerteza da Medição Associada a um Ensaio Aeronáutico em Túnel de Vento Subsônico" [Expression of the Measurement Uncertainty in Aeronautical Subsonic Wind Tunnel Testing], Tese de Doutorado, Universidade Estadual de Campinas, out. de 2000, 103 p.

5 Reis M. L. C. C, Mello O. A. F., Uyeno S., Sampaio O. S. "Calibration Uncertainty of an External Six Component Wind Tunnel Balance”, 
AIAA Paper 2003-3884, $33^{\text {rd }}$ AIAA Fluid Dynamics Conference and Exhibit, 23 - 26 June 2003, Orlando, Florida.

6 BIPM / IEC / IFCC / ISO / IUPAP / OIML, "Guide to the Expression of Uncertainty in Measurements", 1995.

7 Press W. H., Teukolsky S. A., Vetterling W. T., Flannery B. P. Numerical Recipes, Cambridge University Press, 1992.

8 Bevington P. R. Data Redution and Analysis for the Physical Sciences; McGraw-Hill Book Company, 1969.

9 Cox M. G., Daiton M. P., Harris P. M., "Software Suport for Metrology Best Practice Guide n. ${ }^{\circ}$ 6, Uncertainty and Statistical Modelling", Technical report, National Physical Laboratory, Teddington, UK, March 2001.
10 Cox M. G., Forbes A. B., Harris P. M., "Software Suport for Metrology Best Practice Guide n. ${ }^{\circ}$ 4, discrete modelling", Technical report, National Physical Laboratory, Teddington, UK, March 2000, revised January 2002.

11 Barbosa I. M., Hernandez E. M., "Controle Preventivo de Calibrações utilizando Redes Neurais Artificiais com Funções de Base Radiais” Metrologia2003, Brazil.

12 Haykin S., Neural Networks - A comprehensive foundation, Prentice Hall International Inc., 1994.

13 Lira I., Evaluating the measurement uncertainty, IOP, 2003.

14 Hertz J., Anders K., Richard G. P., Introduction to the theory of neural computation, Addison-Wesley Publishing Company, 1995.

\section{ANEXOS:}

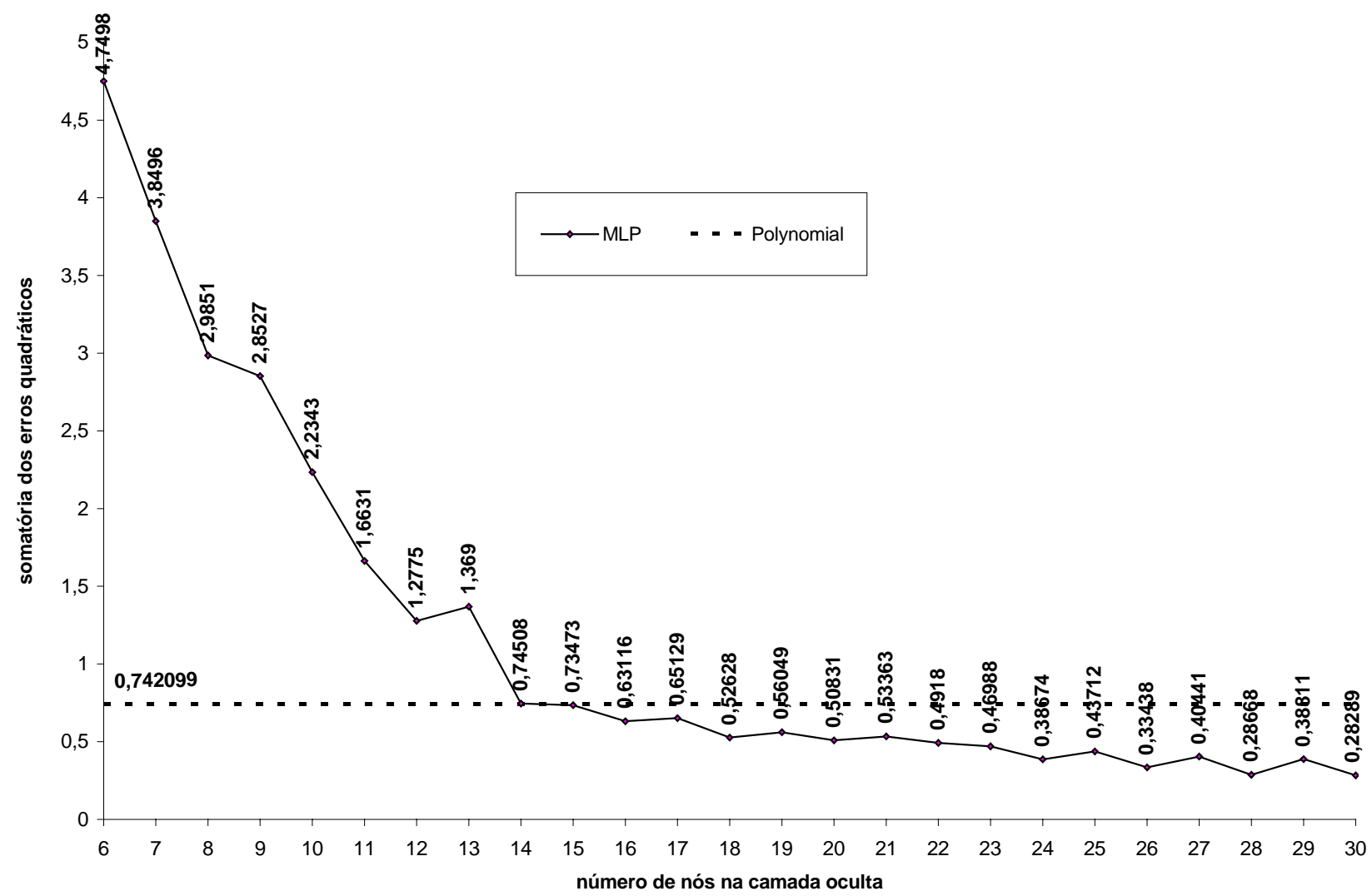

Figura 2 - Comparação da função desempenho $\left(\Sigma e^{2}\right)$ entre 25 Redes Neurais tipo MPL e o polinômio. 
Tabela 2 - Estimativa da função de desempenho total e parcial, $\Sigma e^{2}$, para a Rede Neural e para o polinômio.

\begin{tabular}{|c|c|c|c|c|c|c|c|c|c|c|c|}
\hline Rede & 1 & 2 & 3 & 4 & 5 & 6 & 7 & 8 & 9 & 10 & 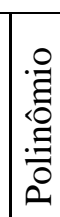 \\
\hline \begin{tabular}{|l|}
$\sum \mathrm{e}^{2}$ \\
Total
\end{tabular} & 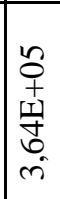 & $\begin{array}{l}\text { L } \\
+ \\
+ \\
\text { t1 } \\
\text { N } \\
\text { - } \\
-1\end{array}$ & 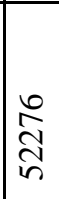 & $\begin{array}{l}\infty \\
\stackrel{\infty}{\stackrel{ \pm}{N}} \\
\underset{m}{ }\end{array}$ & 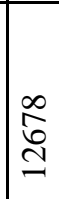 & 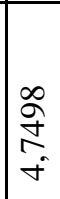 & $\begin{array}{l}0 \\
\text { के } \\
\infty \\
\text { ñ } \\
\dot{n}\end{array}$ & 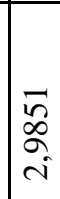 & $\begin{array}{l}\hat{N} \\
\hat{N} \\
\infty \\
\sim\end{array}$ & $\begin{array}{l}\tilde{m} \\
\text { N } \\
\text { vi }\end{array}$ & 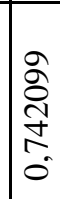 \\
\hline$\sum \mathrm{e}^{2} \mathrm{~F}_{1}$ & $\begin{array}{l}\tilde{\approx} \\
\tilde{\sigma} \\
\tilde{\sigma}\end{array}$ & $\begin{array}{l}0 \\
\tilde{N} \\
\mathscr{\sigma} \\
\sigma\end{array}$ & 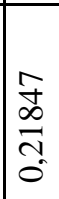 & 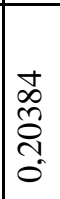 & 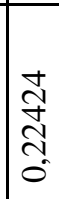 & 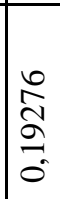 & $\begin{array}{l}\stackrel{M}{\stackrel{N}{N}} \\
\stackrel{-}{0} \\
0\end{array}$ & $\begin{array}{l}\text { के } \\
\text { के } \\
\text { 吕 } \\
\text { ó }\end{array}$ & $\begin{array}{l}\tilde{\mu} \\
0 \\
0 \\
0 \\
0 \\
0\end{array}$ & 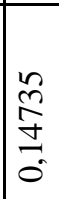 & 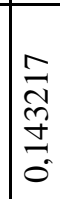 \\
\hline$\Sigma \mathrm{e}^{2} \mathrm{~F}_{2}$ & 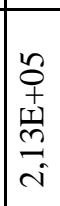 & $\begin{array}{l}\infty \\
0 \\
0 \\
0 \\
0\end{array}$ & $\begin{array}{l}\text { 守 } \\
\text { 足 } \\
6\end{array}$ & $\begin{array}{l}-1 \\
0 \\
0 \\
0\end{array}$ & 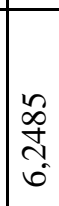 & $\begin{array}{l}0 \\
0 \\
\tilde{N} \\
\hat{\sigma} \\
0 \\
0\end{array}$ & $\mid \begin{array}{l}2 \\
m \\
0 \\
0 \\
0 \\
0 \\
0\end{array}$ & $\begin{array}{l}\hat{\phi} \\
\tilde{\delta} \\
\hat{m} \\
0\end{array}$ & $\begin{array}{l}\tilde{N} \\
\vec{z} \\
\tilde{\sigma}\end{array}$ & 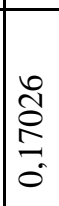 & 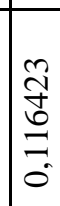 \\
\hline$\Sigma \mathrm{e}^{2} \mathrm{~F}_{3}$ & $\begin{array}{l}n \\
\infty \\
\infty \\
\infty \\
1\end{array}$ & 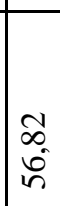 & 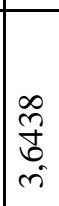 & $\begin{array}{l}\hat{\nu} \\
\hat{n} \\
\tilde{n}^{2}\end{array}$ & 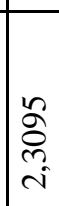 & 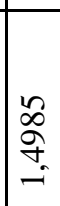 & $\begin{array}{l}0 \\
\infty \\
10 \\
0 \\
\infty \\
0 \\
0\end{array}$ & $\begin{array}{l}\mathfrak{̃} \\
\infty \\
0 \\
15 \\
0 \\
0\end{array}$ & \begin{tabular}{|l}
2 \\
$\infty$ \\
1 \\
$\hat{人}$ \\
0 \\
0 \\
0
\end{tabular} & 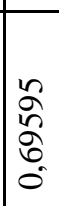 & 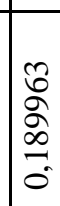 \\
\hline$\sum \mathrm{e}^{2} \mathrm{~F}_{4}$ & $\begin{array}{l}8 \\
\& \\
\stackrel{\circ}{-}\end{array}$ & \begin{tabular}{l}
8 \\
$\infty$ \\
$\infty$ \\
\hdashline
\end{tabular} & $\begin{array}{l}\mathscr{8} \\
\mathscr{8} \\
\stackrel{9}{-}\end{array}$ & $\begin{array}{l}\tilde{\text { }} \\
\stackrel{\sigma}{=} \\
\rightleftharpoons\end{array}$ & 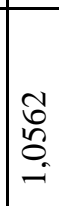 & $\begin{array}{l}\text { Oo } \\
\text { N. } \\
\text { o. } \\
0\end{array}$ & $\begin{array}{l}\text { D } \\
\text { Rे } \\
\text { O. } \\
0\end{array}$ & $\begin{array}{l}\overrightarrow{\tilde{\alpha}} \\
\hat{\sigma} \\
\hat{\sigma}\end{array}$ & $\begin{array}{l}2 \\
0 \\
0 \\
\sigma \\
\sigma \\
0\end{array}$ & $\begin{array}{l}\infty \\
\infty \\
\infty \\
\infty \\
0 \\
0 \\
0\end{array}$ & $\begin{array}{l}\text { N } \\
\hat{\tilde{N}} \\
\hat{\nu} \\
0 \\
0\end{array}$ \\
\hline$\sum \mathrm{e}^{2} \mathrm{~F}_{5}$ & $\begin{array}{l}8 \\
\infty \\
\stackrel{8}{-} \\
\end{array}$ & $\begin{array}{l}8 \\
\infty \\
\infty \\
-\end{array}$ & $\begin{array}{l}\mathscr{8} \\
\stackrel{\infty}{\circ} \\
\stackrel{9}{-}\end{array}$ & 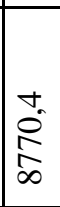 & $\begin{array}{l}\infty \\
\hat{0} \\
\stackrel{0}{0} \\
- \\
-1\end{array}$ & 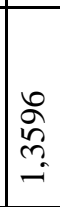 & $\begin{array}{l}\infty \\
m \\
m \\
- \\
-\end{array}$ & $\begin{array}{l}0 \\
0 \\
0 \\
0 \\
0 \\
0 \\
0\end{array}$ & $\begin{array}{l}-\overrightarrow{0} \\
\tilde{0} \\
0 \\
0 \\
0 \\
0\end{array}$ & $\begin{array}{l}10 \\
0 \\
\infty \\
10 \\
0 \\
0\end{array}$ & 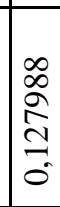 \\
\hline$\sum \mathrm{e}^{2} \mathrm{~F}_{6}$ & 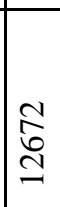 & 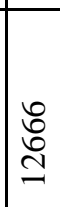 & 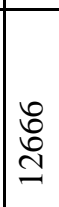 & 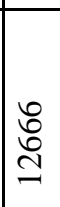 & 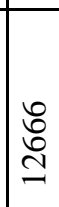 & $\begin{array}{l}\infty \\
\infty \\
0 \\
\stackrel{0}{N} \\
0 \\
0\end{array}$ & 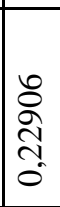 & $\begin{array}{l}\mathcal{N} \\
\hat{N} \\
\mathcal{N} \\
\tilde{o}\end{array}$ & 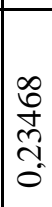 & 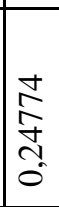 & 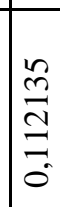 \\
\hline Rede & 11 & 12 & 13 & 14 & 15 & 16 & 17 & 18 & 19 & 20 & \\
\hline \begin{tabular}{|l|}
$\sum \mathrm{e}^{2}$ \\
Total
\end{tabular} & 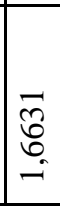 & 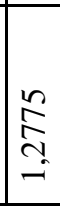 & 量 & 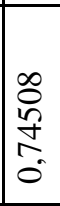 & 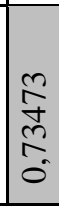 & $\begin{array}{l}0 \\
\tilde{\overrightarrow{\tilde{j}}} \\
0 \\
0\end{array}$ & $\begin{array}{l}\text { तิ } \\
\text { บี } \\
0 \\
0 \\
0\end{array}$ & 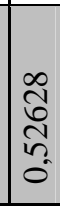 & $\begin{array}{l}9 \\
\text { O } \\
0 \\
10 \\
0 \\
0\end{array}$ & \begin{tabular}{|l}
$\overrightarrow{\tilde{N}}$ \\
0 \\
0 \\
0 \\
0 \\
0
\end{tabular} & 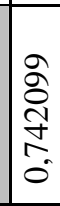 \\
\hline$\overline{\Sigma \mathrm{e}^{2} \mathrm{~F}_{1}}$ & $\mid \begin{array}{l}\hat{\mathscr{Q}} \\
\stackrel{+}{-} \\
\tilde{0}\end{array}$ & 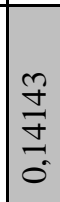 & 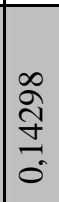 & $\mid \begin{array}{c}N \\
N \\
N \\
0 \\
0\end{array}$ & $\mid \begin{array}{l}\hat{N} \\
\hat{m} \\
\hat{0}\end{array}$ & 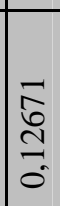 & 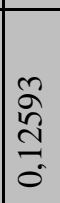 & 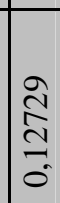 & $\begin{array}{l}\tilde{W} \\
\stackrel{2}{\mathcal{N}} \\
\tilde{0}\end{array}$ & 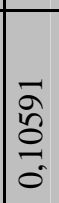 & 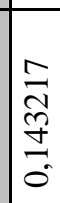 \\
\hline$\Sigma \mathrm{e}^{2} \mathrm{~F}_{2}$ & $\mid$\begin{tabular}{l}
$\tilde{N}$ \\
\multirow{Z}{N}{} \\
$\tilde{O}$
\end{tabular} & 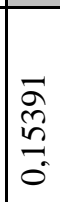 & 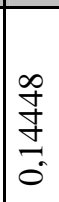 & 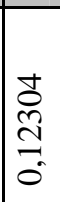 & $\begin{array}{l}0 \\
0 \\
0 \\
= \\
0 \\
0\end{array}$ & $\begin{array}{l}8 \\
\text { - } \\
8 \\
\end{array}$ & $\mid \begin{array}{l}10 \\
\infty \\
0 \\
\infty \\
\infty \\
0 \\
0 \\
0\end{array}$ & $\begin{array}{l}\overrightarrow{\tilde{D}} \\
\tilde{\tilde{ల}} \\
\tilde{0} \\
0\end{array}$ & $\begin{array}{l}0 \\
0 \\
10 \\
0 \\
0 \\
0 \\
0\end{array}$ & $\begin{array}{l}\hat{\widehat{N}} \\
\text { ठ․ } \\
\stackrel{0}{0}\end{array}$ & 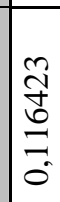 \\
\hline
\end{tabular}




\begin{tabular}{|c|c|c|c|c|c|c|c|c|c|c|c|}
\hline$\Sigma \mathrm{e}^{2} \mathrm{~F}_{3}$ & 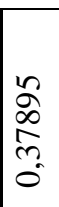 & $\begin{array}{l}\hat{o} \\
o \\
\stackrel{D}{N} \\
\text { ô }\end{array}$ & \begin{tabular}{|l} 
y \\
$\infty$ \\
0 \\
$m$ \\
0 \\
0
\end{tabular} & $\begin{array}{l}\overrightarrow{\tilde{N}} \\
\tilde{D} \\
\infty \\
0 \\
0 \\
0\end{array}$ & $\begin{array}{l}\stackrel{\Omega}{\widehat{N}} \\
\mathcal{N} \\
\tilde{0}\end{array}$ & 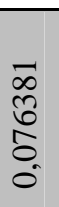 & $\mid \begin{array}{l}m \\
\underset{\sim}{\tilde{N}} \\
\infty \\
0 \\
0 \\
0\end{array}$ & 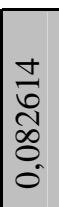 & $\begin{array}{l}\tilde{N} \\
\text { Nิ } \\
\stackrel{0}{0}\end{array}$ & 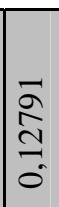 & $\begin{array}{l}\text { ஜ } \\
\mathscr{2} \\
\infty \\
\infty \\
- \\
-\end{array}$ \\
\hline$\sum \mathrm{e}^{2} \mathrm{~F}_{4}$ & $\begin{array}{l}\text { Dे } \\
\mathbb{m} \\
\infty \\
0 \\
0\end{array}$ & $\begin{array}{l}\vec{y} \\
\text { Oे } \\
0 \\
0\end{array}$ & 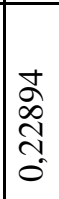 & $\mid \begin{array}{l}\infty \\
\infty \\
\infty \\
\infty \\
\varnothing \\
\varnothing \\
0 \\
0\end{array}$ & 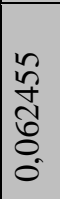 & 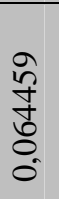 & $\begin{array}{l}\infty \\
\tilde{m} \\
\hat{N} \\
\infty \\
0 \\
0 \\
0\end{array}$ & $\mid \begin{array}{l}\tilde{m} \\
\tilde{\infty} \\
\tilde{\tilde{O}} \\
0 \\
0\end{array}$ & $\begin{array}{l}\tilde{m} \\
\tilde{W} \\
0 \\
\tilde{O} \\
0 \\
0\end{array}$ & $\mid \begin{array}{l}\vec{J} \\
\underset{J}{J} \\
0 \\
0 \\
0\end{array}$ & $\begin{array}{l}\text { N } \\
\hat{\tilde{N}} \\
\underline{\tilde{O}} \\
0\end{array}$ \\
\hline$\sum \mathrm{e}^{2} \mathrm{~F}_{5}$ & 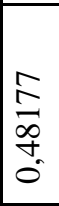 & \begin{tabular}{l}
$\infty$ \\
0 \\
$\tilde{m}$ \\
\multirow{0}{0}{} \\
0
\end{tabular} & \begin{tabular}{|l}
$\hat{y}$ \\
$\overline{8}$ \\
m. \\
0 \\
0
\end{tabular} & 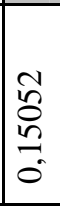 & 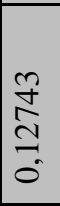 & $\begin{array}{l}m \\
0 \\
0 \\
0\end{array}$ & 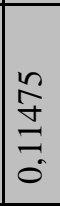 & $\begin{array}{l}\vec{F} \\
\text { ํㅐ } \\
0 \\
0\end{array}$ & 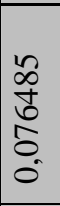 & $\begin{array}{l}9 \\
0 \\
0 \\
0 \\
0 \\
0 \\
0 \\
0\end{array}$ & 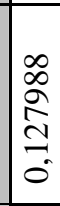 \\
\hline$\overline{\Sigma \mathrm{e}^{2} \mathrm{~F}_{6}}$ & 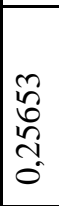 & $\begin{array}{l}\vec{N} \\
\vec{N} \\
\tilde{\Delta} \\
\tilde{0}\end{array}$ & 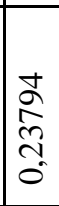 & $\begin{array}{l}1 \\
0 \\
L \\
\infty \\
0 \\
0 \\
0\end{array}$ & 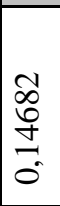 & $\begin{array}{l}\overrightarrow{\mathscr{D}} \\
\stackrel{m}{0} \\
\overrightarrow{0}\end{array}$ & 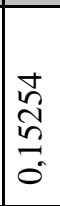 & 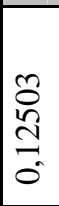 & 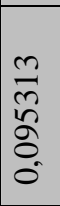 & 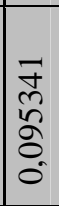 & 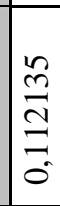 \\
\hline Rede & 21 & 22 & 23 & 24 & 25 & 26 & 27 & 28 & 29 & 30 & \\
\hline \begin{tabular}{|l|}
$\sum \mathrm{e}^{2}$ \\
Total
\end{tabular} & $\begin{array}{l}\tilde{y} \\
\hat{n} \\
\tilde{\omega} \\
\hat{\omega} \\
0 \\
0\end{array}$ & \begin{tabular}{|l}
$\infty$ \\
\\
\\
\\
0 \\
\end{tabular} & \begin{tabular}{|l}
$\infty$ \\
$\infty$ \\
0 \\
0 \\
0 \\
0 \\
0 \\
0
\end{tabular} & \begin{tabular}{|l}
1 \\
0 \\
$\infty$ \\
0 \\
0 \\
0 \\
0 \\
\end{tabular} & 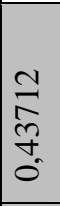 & 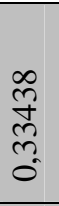 & 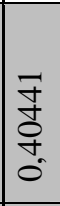 & \begin{tabular}{|l}
$\infty$ \\
0 \\
0 \\
$\infty$ \\
$\sim$ \\
0 \\
0 \\
\end{tabular} & $\begin{array}{l}\vec{D} \\
\infty \\
\infty \\
m \\
0 \\
0\end{array}$ & 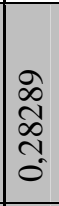 & 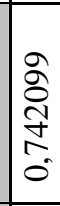 \\
\hline$\sum \mathrm{e}^{2} \mathrm{~F}_{1}$ & $\begin{array}{l}0 \\
0 \\
0 \\
0 \\
0\end{array}$ & $\begin{array}{l}\infty \\
0 \\
0 \\
0 \\
0 \\
0 \\
0\end{array}$ & 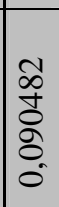 & $\begin{array}{l}\text { ڤి } \\
\stackrel{2}{ } \\
\infty \\
\infty \\
0 \\
0\end{array}$ & 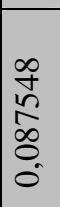 & 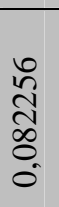 & $\begin{array}{l}\text { बे } \\
\text { ते } \\
0 \\
0 \\
0 \\
0\end{array}$ & $\mid \begin{array}{l}2 \\
0 \\
0 \\
0 \\
0 \\
0\end{array}$ & $\begin{array}{l}0 \\
\infty \\
\overrightarrow{7} \\
\overrightarrow{0} \\
0 \\
0 \\
0\end{array}$ & 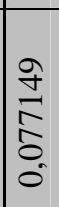 & 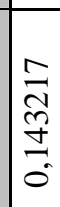 \\
\hline$\sum \mathrm{e}^{2} \mathrm{~F}_{2}$ & 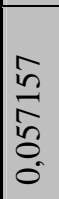 & 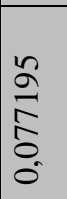 & 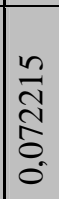 & $\begin{array}{l}8 \\
0 \\
\infty \\
o 0 \\
8 \\
0 \\
0\end{array}$ & $\begin{array}{l}2 \\
0 \\
\infty \\
\mathscr{D} \\
\qquad \\
0 \\
0\end{array}$ & 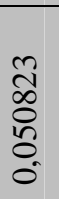 & $\begin{array}{l}\overrightarrow{\tilde{D}} \\
\overline{8} \\
\bar{\delta} \\
0 \\
0\end{array}$ & 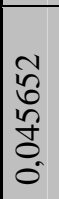 & $\begin{array}{l}m \\
\hat{0} \\
0 \\
\text { L } \\
0 \\
0\end{array}$ & 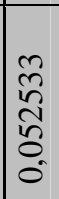 & 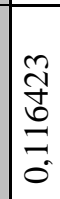 \\
\hline$\Sigma \mathrm{e}^{2} \mathrm{~F}_{3}$ & $\begin{array}{l}0 \\
0 \\
0\end{array}$ & $\begin{array}{l}\underset{J}{J} \\
\underset{0}{J} \\
0 \\
0\end{array}$ & $\begin{array}{l}8 \\
0 \\
10 \\
0 \\
0 \\
0\end{array}$ & 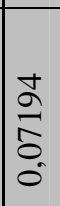 & 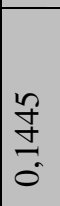 & 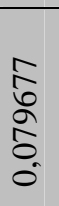 & 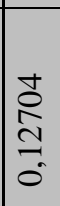 & $\mid \begin{array}{l}\vec{J} \\
\widetilde{N} \\
\hat{\widetilde{a}} \\
0 \\
0\end{array}$ & $\begin{array}{l}\overrightarrow{\hat{N}} \\
\stackrel{m}{0} \\
\stackrel{0}{0}\end{array}$ & 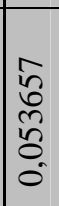 & $\begin{array}{l}\text { ஜ } \\
\mathscr{2} \\
\infty \\
\infty \\
-1 \\
0\end{array}$ \\
\hline$\Sigma \mathrm{e}^{2} \mathrm{~F}_{4}$ & 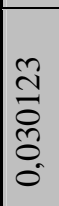 & 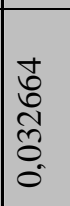 & 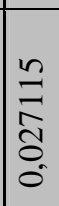 & 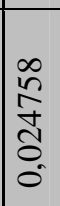 & $\begin{array}{l}0 \\
0 \\
0 \\
0 \\
0 \\
0 \\
0\end{array}$ & $\begin{array}{l}\hat{f} \\
0 \\
0 \\
0 \\
0\end{array}$ & 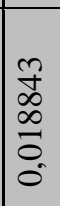 & $\begin{array}{l}\hat{\sigma} \\
\sigma \\
\hat{N} \\
0 \\
0 \\
0\end{array}$ & 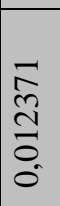 & 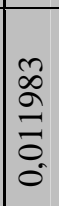 & $\begin{array}{l}\mathcal{N} \\
\hat{\tilde{N}} \\
\underline{0} \\
0 \\
0\end{array}$ \\
\hline$\sum \mathrm{e}^{2} \mathrm{~F}_{5}$ & $\begin{array}{c}10 \\
\stackrel{10}{0} \\
0 \\
0 \\
0\end{array}$ & 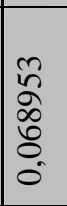 & $\begin{array}{l}\text { - } \\
0 \\
0 \\
0 \\
0 \\
0 \\
0\end{array}$ & $\begin{array}{l}\infty \\
0 \\
\delta \\
\delta \\
8 \\
0 \\
0\end{array}$ & $\begin{array}{l}\tilde{J} \\
\exists \\
\bar{\sigma} \\
0 \\
0\end{array}$ & $\begin{array}{l}\infty \\
0 \\
\mathscr{2} \\
\infty \\
0 \\
0 \\
0 \\
0\end{array}$ & 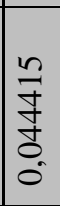 & 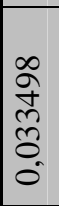 & 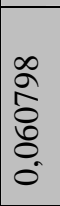 & 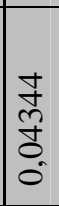 & 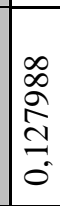 \\
\hline$\Sigma \mathrm{e}^{2} \mathrm{~F}_{6}$ & $\begin{array}{l}m \\
m \\
\stackrel{9}{\delta} \\
\dot{O} \\
0 \\
0\end{array}$ & $\begin{array}{l}\hat{O} \\
\mathbb{N} \\
0 \\
0 \\
0 \\
0\end{array}$ & $\mid \begin{array}{l}\mathscr{2} \\
\infty \\
\vec{\Xi} \\
0 \\
0 \\
0\end{array}$ & \begin{tabular}{l} 
م \\
$\hat{N}$ \\
\multirow{1}{\hat{O}}{} \\
0 \\
0
\end{tabular} & $\begin{array}{l}\text { ते } \\
\widetilde{\widetilde{U}} \\
\text { ठ. } \\
0\end{array}$ & $\begin{array}{l}\text { Wै } \\
\text { मू } \\
0 \\
0\end{array}$ & $\mid \begin{array}{l}0 \\
\infty \\
0 \\
\hat{-} \\
0 \\
0 \\
0 \\
0\end{array}$ & 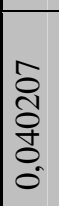 & $\begin{array}{l}m \\
\text { D. } \\
\hat{N} \\
0 \\
0 \\
0\end{array}$ & 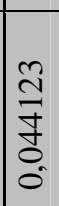 & 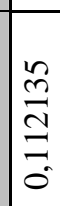 \\
\hline
\end{tabular}


Tabela 3: resultados do GUM e MSC (1000000 histórias) para a incerteza nas cargas aerodinâmicas. Unidade: newton para força e newton×metro para momento).

\begin{tabular}{|c|c|c|c|c|c|c|}
\hline \multirow[t]{2}{*}{$F i$} & \multirow{2}{*}{$\begin{array}{c}\text { Carreganent } \\
\text { o n. } .^{\circ}\end{array}$} & \multirow[t]{2}{*}{ Fi aplicado } & \multicolumn{2}{|c|}{ Fi ajustado } & \multicolumn{2}{|c|}{$u(F i)$} \\
\hline & & & GUM & $M S C$ & GUM & $M S C$ \\
\hline \multirow[t]{5}{*}{$F_{1}$} & 1 & 0 & 0.51195 & 0.51197 & 0.38407 & 0.38450 \\
\hline & 26 & 500 & 499.16293 & 499.16270 & 0.28691 & 0,28684 \\
\hline & 38 & 0 & -0.41340 & -0.41290 & 0.35429 & 0.35467 \\
\hline & 60 & 500 & 499.25639 & 499.25697 & 0.28653 & 0.28656 \\
\hline & 71 & 1000 & 999.98652 & 999.98572 & 0.41589 & 0.41536 \\
\hline \multirow[t]{5}{*}{$F_{2}$} & 1 & 0 & -0.20259 & -0.20268 & 0.38152 & 0.38151 \\
\hline & 26 & 0 & -0.10234 & -0.10278 & 0.30425 & 0.30431 \\
\hline & 38 & 800 & 800.10258 & 800.10211 & 0.35734 & 0.35752 \\
\hline & 60 & 0 & 0.11840 & 0.11862 & 0.30405 & 0.30418 \\
\hline & 71 & 0 & -0.22420 & -0.22428 & 0.40774 & 0.40727 \\
\hline \multirow[t]{5}{*}{$F_{3}$} & 1 & -2400 & -2400.12430 & -2400.12419 & 1.16578 & 1.16690 \\
\hline & 26 & 0 & 0.25197 & 0.25176 & 1.12808 & 1.12838 \\
\hline & 38 & 0 & 0.03492 & 0.03396 & 1.15320 & 1.15320 \\
\hline & 60 & 0 & 0.21573 & 0.21709 & 1.12800 & 1.13008 \\
\hline & 71 & 2400 & 2399.50371 & 2399.50211 & 1.18017 & 1.17960 \\
\hline \multirow[t]{5}{*}{$F_{4}$} & 1 & 0 & -0.51217 & -0.51234 & 0.28400 & 0.28318 \\
\hline & 26 & -300 & -300.32613 & -300.32627 & 0.23836 & 0.23855 \\
\hline & 38 & 0 & -0.07717 & -0.07781 & 0.26948 & 0.27035 \\
\hline & 60 & 0 & -0.14593 & -0.14584 & 0.23825 & 0.23808 \\
\hline & 71 & 0 & -0.34989 & -0.34944 & 0.29994 & 0.30099 \\
\hline \multirow[t]{5}{*}{$F_{5}$} & 1 & -300 & -299.76879 & -299.76868 & 0.36256 & 0.36245 \\
\hline & 26 & 0 & -0.07098 & -0.07091 & 0.27053 & 0.27057 \\
\hline & 38 & 0 & -0.66202 & -0.66137 & 0.33436 & 0.33455 \\
\hline & 60 & -300 & -300.31701 & -300.31727 & 0.27029 & 0.27005 \\
\hline & 71 & 0 & -0.30006 & -0.29976 & 0.39268 & 0.39189 \\
\hline \multirow[t]{5}{*}{$F_{6}$} & 1 & 0 & 0.01674 & 0.16440 & 0.33987 & 0.34002 \\
\hline & 26 & 0 & 0.37475 & 0.37458 & 0.25391 & 0.25448 \\
\hline & 38 & 240 & 240.57584 & 240.57637 & 0.31353 & 0.31326 \\
\hline & 60 & 0 & 0.25371 & 0.25390 & 0.25368 & 0.25416 \\
\hline & 71 & 0 & 0.13969 & 0.13991 & 0.36802 & 0.36863 \\
\hline
\end{tabular}

